ENCYCLOPÉDIE Encyclopédie berbère

BERBERE

10 | 1991

10 | Beni Isguen - Bouzeis

\title{
Beni Snassen / Beni iznasen (en berb. : At Iznasn)
}

\author{
E.B. et S. Chaker
}

\section{OpenEdition}

Journals

Édition électronique

URL : http://journals.openedition.org/encyclopedieberbere/1685

DOI : 10.4000/encyclopedieberbere.1685

ISSN : 2262-7197

\section{Éditeur}

Peeters Publishers

\section{Édition imprimée}

Date de publication : 1 décembre 1991

Pagination : 1468-1470

ISBN : 2-85744-549-0

ISSN : 1015-7344

Référence électronique

E.B. et S. Chaker, «Beni Snassen / Beni iznasen (en berb. : At Iznasn)», Encyclopédie berbère [En ligne], $10 \mid$ 1991, document B63, mis en ligne le 01 mars 2013, consulté le 25 septembre 2020. URL : http:// journals.openedition.org/encyclopedieberbere/1685; DOI : https://doi.org/10.4000/ encyclopedieberbere.1685

Ce document a été généré automatiquement le 25 septembre 2020.

(c) Tous droits réservés 


\title{
Beni Snassen / Beni iznasen (en berb. : At Iznasn)
}

\author{
E.B. et S. Chaker
}

\section{Les monts des Beni Snassen (E.B.)}

1 Petite chaîne calcaire dans la région tellienne du Maroc oriental qui doit son nom à un groupe berbère zénète. Il s'agit d'un pli unique dissymétrique de calcaire dolomitique dont le noyau est constitué de schistes primaires. Il culmine à $1535 \mathrm{~m}$ au Ras Fourhal. Une orogénie tertiaire, particulièrement vigoureuse en bordure de la fosse méditerranéenne, est responsable de cette chaine abrupte sur la face nord qui domine la plaine de Zébra et de Berkane; la pente vers le sud-sud-ouest est plus régulière ; ce qui n'empêche pas le voyageur de subir le contraste très vif entre ce versant du massif et les plaines de caractère steppique du Maroc oriental, que ce soit celle de la Moulouya ou celle des Angads. Cette opposition est autant d'ordre climatique que topographique. L'augmentation de la pluviosité (600 $\mathrm{mm}$ au sommet contre $350 \mathrm{~mm}$ à Oujda) et l'humidité de l'air permettent des cultures florissantes arrosées de nombreuses séguias alimentées par des sources abondantes. Cette plus forte humidité favorise les boisements de chênes verts, de thuyas et de pins d'Alep, plus vigoureux sur le versant nord que sur le versant sud.

2 Partout les Beni Snassen cultivent des amandiers, des oliviers et des figuiers qui bordent des céréales ou des légumes de plein champ; dans les vallées et les plaines de Zébra et de Berguent dominent les orangers et autres agrumes très réputés. Les villages en pierres sèches, parfois importants, occupent, dans le massif des positions pittoresques qui justifient en partie l'appellation de nids d'aigle qu'emploient avec quelque exagération les guides touristiques. La forêt dégradée en partie souffre du parcours des troupeaux dont c'est le lieu de pacage traditionnel et encore plus de la pression démographique. Cette chaîne de moins de $50 \mathrm{~km}$ de longueur est peuplée de plus de 100000 habitants, ce qui lui donne une densité de 40 habitants au $\mathrm{km}^{2}$. Le trop plein de main d'œuvre se déverse dans les plaines du nord et du sud et contribue à une 
importante migration dans les villes (Oujda, Fès, Casablanca) et l'Europe. Pendant l'époque coloniale, comme tous les Rifains, les Beni Snassen fournissaient une main d'œuvre recherchée par les grandes exploitations agricoles de l'Algérie occidentale, particulièrement pour la taille des oliviers et de la vigne.

3 La chaîne est traversée par une route qui rejoint el-Aïoun à Berkane, son parcours est pittoresque, surtout dans la zone dolomitique; elle passe à proximité de l'important gisement préhistorique de la grotte des Pigeons, à Taforalt*; à l'ouest de ce bourg commencent les gorges du Zeggel qu'emprunte une voie secondaire qui permet également de rejoindre Berkane; mais la route principale, d'Oujda à Berkane, contourne le massif par l'ouest.

\section{Les Beni Iznasen. Linguistique (S. Chaker)}

Cette confédération de tribus berbérophones, située à l'est de la zone dite "rifaine " (entre la frontière algérienne et le reste du bloc rifain) est comprise dans un triangle délimité à l'est par l'Oued Kiss et à l'ouest par la Moulouya.

5 Selon Renisio (1932), les sous-groupes constitutifs de la confédération sont les :

- At Khaled- At Menquš

- At Ahtiq

- At Urimmeš

6 Au plan linguistique, les B.I. appartiennent clairement à l'ensemble rifain, avec cependant un caractère nettement moins accusé des évolutions phonétiques propres au reste de ce domaine dialectal ; ce qui amène de nombreux auteurs (anciens) à les en distinguer (Renisio notamment) ; la tendance à la confusion $/ 1 /-/ r /$, à la vocalisation de $/ \mathrm{r}$ / et à la palatalisation $(/ \mathrm{ll} /$ > / /g//), typiques du rifain "standard", n'y sont pas attestées. A ce point de vue, le parler des B.I. est donc plus proche de ses voisins du sud/sud-est, extérieurs à la zone rifaine: Beni Snous (en Algérie), Ayt Warayn, Ayt Seghrouchen. La chute de la voyelle initiale des noms masculins y est également plus rare. La spirantisation par contre y est très avancée et touche :

- les dentales: / $t /$, normalement réalisé $[t]$, évolue parfois même jusqu'au souffle laryngal [h] (nihnin < nitnin « eux »);/d/ est normalement réalisé [d] (zdem « ramasser le bois »);

- les palato-vélaires: / $\mathrm{k} /$ est régulièrement traité en [k] (aksum $<a k s u m) ; / \mathrm{g} />\mathrm{y}>\mathrm{i}$ (sans doute long [i:]) (isegres < isi:res « mangeoire », asegnu > asi:nu « grosse aiguille »...

- à l'inverse, /y/, surtout en position implosive, évolue souvent vers /š/ (ayt > ašt). Une étude spécifique a récemment été consacrée au phénomène de spirantisation dans ce parler (Elkirat, 1987).

7 Traditionnellement classé avec le rifain dans la catégorie des dialectes «zénètes ", le berbère des B.I. présente, comme tous les parlers de cet hypothétique ensemble, un thème verbal spécifique d'aoriste intensif négatif dont l'extension a bien été mise en évidence par Kossman (1989).

8 Étymologie de l'ethnonyme : le second élément du nom des B.I. s'intègre parfaitement dans le modèle onomastique général que j'ai proposé il y a quelques années (Chaker, 1983) : segment verbal ou nominal + affixe personnel (en l'occurrence -sn, $3^{\mathrm{e}}$ personne du masculin pluriel $=$ « leur, à eux »). L'initiale $i$ - du premier segment peut être analysée soit comme l'indice de $3^{\mathrm{e}}$ personne du masculin singulier d'un verbe, soit comme 
marque initiale de pluriel nominal; il est donc difficile de décider de l'identité syntaxique de ce premier élément izna qui peut être aussi bien un verbe (au thème de prétérit ; radical *ZN(?) ; d'où : izna-sn.= « il leur a... »), qu'un nom masculin pluriel (d'où izna-sn $=$ «leur... »).

9 Outre les quelques références spécifiques aux B.I., on se reportera donc à l'ensemble de la bibliographie linguistique consacrée au Rif.

\section{BIBLIOGRAPHIE}

Basset R., « Notice sur le dialecte berbère des Beni Iznacen », Giornale della societa asiatica italiana, t. 11, 1898, pp. 1-14.

Basset R., Loqman berbère, Paris, 1890 (un texte B.I.).

Chaker S., " Onomastique berbère ancienne (Antiquité-Moyen Age) : rupture et continuité », B.C.T.H., 19 (B), 1983, pp. 483-397 ; repris dans Textes en linguistique berbère..., Paris, Éditions du CNRS, 1984 (chap. 14).

Destaing E., Dictionnaire français-berbère (dialecte des Beni Snous), [1308 notations B.I.], Paris, Leroux, 3 vol. 1907-1914.

Elkirat Y., Spirantization in the Beni Iznasen Dialect. Diachrony and Synchrony, DES linguistique, Faculté des Lettres de Rabat, 1987.

Kossman M., «L'inaccompli négatif en berbère », Etudes et documents berbères, t. 6, 1989, pp. 19-29.

Renisio A., Etude sur les dialectes berbères des Beni Iznassen, du Rif et des Senhaja de Sraïr..., Paris, Leroux, 1932, 465 p. (référence la plus importante sur les B.I. : phonétique, grammaire, textes et lexique).

INDEX

Mots-clés : Géographie, Tribus 